\title{
Ginsenoside Rb1 Improves Brain, Lung, and Intestinal Barrier Damage in Middle Cerebral Artery Occlusion/Reperfusion (MCAO/R) Mice Through the PPARY Signaling Pathway
}

zhuo Chen

China Pharmaceutical University

shuaishuai Gong

China Pharmaceutical University

Yuchuan Ren

China Pharmaceutical University

jieman wang

China Pharmaceutical University

Fang Li

China Pharmaceutical University

YuanYuan Zhang

China Pharmaceutical University

Junping Kou ( $\nabla$ junpingkou@cpu.edu.cn )

China Pharmaceutical University https://orcid.org/0000-0001-8377-915X

\section{Research}

Keywords: Focal ischemic stroke, brain/lung/intestinal barriers, ginsenoside Rb1, PPARY

Posted Date: January 12th, 2021

DOI: https://doi.org/10.21203/rs.3.rs-141957/v1

License: (c) (1) This work is licensed under a Creative Commons Attribution 4.0 International License. Read Full License 


\section{Abstract}

\section{Background}

Ischemic stroke causes brain inflammation and multi-organ injury, in which the peroxisome proliferatoractivated receptor-gamma (PPARY) signaling pathway plays a key role. Studies have indicated that ginsenoside Rb1 (GRb1) can protect blood-brain barrier integrity after stroke. Here, we aimed to determine whether GRb1 can ameliorate brain/lung/intestinal barrier damage in mice with acute cerebral ischemia through the PPARy signaling pathway.

Method

Adult male ICR (Institute of Cancer Research) mice underwent right middle cerebral artery occlusion for 1 $\mathrm{h}$ with subsequent $24 \mathrm{~h}$ reperfusion to produce ischemia/reperfusion(I/R) injury, following which the mice were administered GRb1 $(20 \mathrm{mg} / \mathrm{kg})$ intraperitoneally. The integrity of the brain, pulmonary, and intestinal barriers was assessed using an Evans blue leakage assay and connexin immunofluorescence staining. The serum levels of tumor necrosis factor-a (TNF-a) and interferon-gamma (IFN- $\gamma$ ) were measured by enzyme-linked immunosorbent assay (ELISA), and the expression of PPARY and phospho-nuclear factor kappa B (NF-KB) p65 was determined by western blot.

Result

GRb1 significantly mitigated multi-organ injury and increased the expression of cerebral microvascular, pulmonary vascular, and intestinal epithelial connexins (claudin-5, occludin, and zona occludens 1 (ZO-1). In brain, lung, and intestinal tissues, GRb1 activated PPARy, decreased phospho-NF-kB p65 levels, and inhibited the production of proinflammatory cytokines, thereby maintaining barrier permeability. However, cotreatment with GRb1 and the PPARy antagonist GW9662 reversed the barrier-protective effect of GRb1.

Conclusion

The results showed that GRb1 can improve brain/lung/intestinal barrier damage post-stroke through the PPARY pathway.

\section{Highlights}

1. Pulmonary and intestinal barriers are disrupted simultaneously in MCAO/R mice.

2. PPARY plays an important role in maintaining the integrity of multi-organ barriers.

3. GRb1 maintains the integrity of multi-organ barriers through the PPARy signaling pathway.

\section{Introduction}

Stroke accounts for almost $5 \%$ of all disability-adjusted life years (DALYs)[1] and $10 \%$ of all deaths worldwide[2], with the bulk of this burden (over $75 \%$ of deaths from stroke and $81 \%$ of DALYs) falling on 
low- and middle-income countries[3]. It has become increasingly clear that stroke causes multi-organ systemic diseases and triggers systemic responses[4]. Pulmonary infection and stress ulceration are the most common complications for severe stroke patients[5].

Several studies have focused on stroke-induced lung infection. The lung is particularly vulnerable after stroke, including ischemic and hemorrhagic strokes[6, 7]. Elmer[8] et al. reported that $15.6 \%$ of stroke patients develop acute lung injury(ALI) within $36 \mathrm{~h}$ of hospital admission, while $7.8 \%$ develop pneumonia or bronchitis during hospitalization. Current clinical studies frequently report that CNS injury patients develop gut microbiota dysbiosis, gut dysmotility, gastrointestinal bleeding, and even gut-origin sepsis, effects that are regularly associated with worse outcomes, including deteriorating functional deficits and increased mortality rates[9]. After an ischemic and hemorrhagic stroke, approximately $50 \%$ of patients experience gastrointestinal complications, including dysphagia, gastrointestinal hemorrhaging, constipation, and bowel incontinence[10,11]. Furthermore, gut permeability has been shown to increase after stroke in mice, and correlates with stroke severity[12]. However, there is a lack of systematic and indepth research on the role and mechanism underlying the effects of acute ischemic stroke on the pulmonary and intestinal barriers.

Peroxisome proliferator-activated receptor-gamma (PPARY), a stress-induced transcription factor, plays essential roles in the regulation of cellular differentiation, development, and metabolism[13]. Numerous studies have shown that PPARy plays a key role in the barrier functions of epithelial and endothelial cells. PPARy maintains the integrity of the blood-brain barrier (BBB) via inhibiting hypoxia-induced nuclear factor kappa B (NF-KB) nuclear translocation[14]. It has been shown that magnolol can mitigate lipopolysaccharide (LPS)-induced ALI, and this is achieved, at least partly, through PPARY activation[15]. There is also convincing evidence that the microbiota influences intestinal PPARY in maintaining intestinal mucosal homeostasis[16].

Panax ginseng (Renshen, Chinese ginseng) is a traditional Chinese herbal medicine that is extensively used worldwide as a medicinal and nutritional supplement for a variety of disorders $[17,18]$. More than 40 different ginsenosides have been isolated from ginseng[19], including GRb1, which was identified as one of its main active ingredients[20]. GRb1 has been reported to play a neuroprotective role in the acute phase of cerebral ischemia by protecting BBB integrity, increasing regional cerebral blood flow, and enhancing the stability of the neuronal ultrastructure[21]. Moreover, GRb1 can significantly attenuate LPSinduced lung microcirculatory injury[22], and can protect the intestinal mucosal barrier against damage induced by peritoneal air exposure[23]. Recent studies have demonstrated that GRb1 promotes adipogenesis through the regulation of PPARY[24] and is a potential ligand of PPARY, as evidenced via a newly developed method called polarized total internal reflection fluorescence microscopy (poITIRFM) [25]. Despite the large number of reports on the wide-ranging effects of GRb1, whether this ginsenoside can alleviate multi-organ injury induced by ischemic stroke via PPARY remains unknown. Therefore, in the present study, we investigated how GRb1 affects the brain/lung/intestinal barriers using a middle cerebral artery occlusion/reperfusion (MCAO/R) mouse model, and explored the putative underlying mechanisms. 


\section{Materials And Methods \\ 2.1. Reagents}

GRb1 (CAS number 41753-43-9) with a purity $>98 \%$ was purchased from Aladdin Biochemical Technology (Shanghai, China). Other chemical agents used in this study were of at least analytical grade. Edaravone(Eda), an intravenous medication used to help with recovery following a stroke and also to treat amyotrophic lateral sclerosis, was purchased from Simcere Pharmaceutical Group (Nanjing, China). LPS from Escherichia coli strain 0111:B4 purified by phenol extraction was purchased from SigmaAldrich (Shanghai, China). Colitis-grade dextran sulfate sodium (DSS; 36,000-50,000 MW) was purchased from MP Biomedicals (Shandong, China). GW9662 (CAS number: 22978-25-2), a selective PPAR antagonist with an $\mathrm{IC}_{50}$ of $3.3 \mathrm{nM}$, was purchased from Aladdin Biochemical Technology with a purity $>98 \%$.

\subsection{Animals}

One hundred and thirty-two male ICR (Institute of Cancer Research) mice (6-8 weeks old) were provided by the Comparative Medicine Center of Yangzhou University (Yangzhou, China; certificate no. SCXK 20190024). All the procedures and assessments were approved by the Animal Ethics Committee of the School of Chinese Materia Medica, China Pharmaceutical University. The experiments were carried out in accordance with the National Institutes of Health Guide for the Care and Use of Laboratory Animals (National Institutes of Health Publication No. $80-23$, revised in 1996). The animals were housed in a controlled environment $\left(12: 12 \mathrm{~h}\right.$ light/dark cycle, humidity of $60 \pm 5 \%$, and a temperature of $\left.22 \pm 3{ }^{\circ} \mathrm{C}\right)$ and were allowed access to food and water ad libitum. Before surgery, all the mice were allowed to acclimatize to the laboratory conditions for at least three days. All the measurements of outcome were performed by investigators who were blinded to the experimental conditions.

2.3. Preparation of the cerebral ischemia/reperfusion (I/R) model, and induction of acute colitis, and the mouse model of ALI

$\mathrm{MCAO} / \mathrm{R}$ was performed by intraluminal nylon filament intrusion in accordance with Longa's method[26]. Briefly, mice were anesthetized by intraperitoneal injection of $3 \%$ pentobarbital sodium $(0.1 \mathrm{~mL} / 10 \mathrm{~g}$ body weight); then, the neck vessels were exposed by a midline incision and the branches of the right external carotid artery were carefully isolated and cauterized. A 6-0 nylon monofilament suture, blunted at the tip and coated with $1 \%$ poly-L-lysine, was advanced 9-10 $\mathrm{mm}$ into the internal carotid to occlude the origin of the middle cerebral artery. During surgery and ischemia, the temperature of the animal was maintained at $37.0 \pm 0.5^{\circ} \mathrm{C}$ with a heating pad (ALCBIO, Shanghai, China). After $60 \mathrm{~min}$ of occlusion, reperfusion was achieved by the withdrawal of the suture. The same treatment was carried out on mice in the Sham group, except that the suture was not advanced into the internal carotid artery[27]. A laser Doppler flowmeter (FLPI2, Moor Instruments Ltd, Axminster, UK) was used to confirm that middle cerebral artery blood flow had decreased to $<30 \%$ of the basic cerebral blood flow immediately after the occlusion. Animals with a blood flow of less than $30 \%$ that of preischemic levels were used for further study. 
Acute colitis was induced by DSS $(3 \%, w / v)$ administered in double-distilled drinking water for seven consecutive days[28].

ALI was induced by intratracheal instillation of LPS ( $5 \mathrm{mg} / \mathrm{kg}, 0.9 \%$ saline). The mice were euthanized $6 \mathrm{~h}$ after LPS challenge[29].

\subsection{Groups}

Ninety-six ICR mice were randomly divided into the following six groups ( $n=16$ per group) according to the random number table generated by SPSS software 22.0 (SPSS Inc., Chicago, IL, USA): i) Sham group, where the mice received a sham operation and an equal volume of normal saline; ii) Model group, where the mice received MCAO/R and an equal volume of normal saline; iii) GRb1 group, where the mice received MCAO/R and were treated with $20 \mathrm{mg} / \mathrm{kg}$ GRb1 (dissolved in normal saline) administered by intraperitoneal injection after $1 \mathrm{~h}$ of ischemia; iv) Eda group, where the mice received MCAO/R and were treated with $3 \mathrm{mg} / \mathrm{kg}$ Edaravone (dissolved in normal saline) administered by intraperitoneal injection after $1 \mathrm{~h}$ of ischemia; v) DSS group, where the mice received 3\% DSS for seven days; vi) LPS group, where the mice received $5 \mathrm{mg} / \mathrm{kg}$ LPS administered intratracheally. Subsequently, the mice were administrated with GRb1 with or without GW9662. ICR mice $(n=36)$ were randomly divided into the following six groups: Sham group, Model group, GRb1 group (Model + GRb1), GW9662 plus Sham group (Sham + GW), GW9662 plus Model group (Model + GW), and GRb1 plus GW9662 plus Model group (Model + GRb1 + $\mathrm{GW}$ ). One hour before surgery, mice from the Sham $+\mathrm{GW}$, Model $+\mathrm{GW}$, and Model + GRb1 + GW groups were intraperitoneally injected with GW9662 (4 mg/kg body weight).

\subsection{2,3,5-Triphenyltetrazole ammonium chloride (TTC) staining}

Brains were quickly removed at $24 \mathrm{~h}$ after reperfusion. The 2, 3, 5-triphenyl tetrazolium chloride (TTC) staining was performed to evaluate tissue viability and measure the infarct size. The infarct area was measured in NIH Image $\mathrm{J}$ software (Version 1.42; National Institutes of Health, Bethesda, Md). The infarct areas on each slice were summed and multiplied by slice thickness to give the infarct volume. Infarct volume was expressed as a percentage of infarction per ipsilateral hemisphere[30].

\subsection{Determination of Evans blue (EB) leakage in the brain, lung, and small intestine}

As previously described[31], $2 \mathrm{~h}$ before the mice were euthanized ( $n=6$ per group), $2 \%$ EB (Sigma-Aldrich, St. Louis, MO, USA) in normal saline was injected into mice through the tail vein. After $2 \mathrm{~h}$, the mice were anesthetized and perfused with $0.9 \%$ normal saline. The brains, lungs, and small intestines were taken from the mice and photographed. The brain, lungs, and intestines were dried with filter paper, weighed, and frozen in liquid nitrogen. Tissue homogenates were prepared (100 $\mathrm{mg}$ tissue/ $\mathrm{mL}$ formamide), incubated at $60{ }^{\circ} \mathrm{C}$ for $18 \mathrm{~h}$, and centrifuged at $5,000 \times g$ for $30 \mathrm{~min}$. The absorbance of the tissue supernatants was measured using a microplate spectrophotometer at $620 \mathrm{~nm}$. The EB content was calculated according to an EB standard curve. 


\subsection{Changes in mesenteric blood flow}

After anesthesia with $3 \%$ pentobarbital sodium, an incision of about $1-2 \mathrm{~cm}$ was made in the abdominal cavity of mice. Blood flow in the mesentery was measured using a laser Doppler flowmeter[32].

\subsection{Neutrophil count in bronchoalveolar lavage fluid (BALF)}

The lung lumen was sampled by flushing with $500 \mu \mathrm{L}$ of PBS using a syringe. The BALF was centrifuged at $1500 \mathrm{rpm}$ for $5 \mathrm{~min}$ at $4{ }^{\circ} \mathrm{C}$ and the supernatant was collected for further analysis[33].

\subsection{Histomorphological analysis}

Mice ( $n=3$, for each group) were sacrificed after MCAO/R, and transcardially perfused with physiological saline from the heart to the systemic circulation until the liver appeared white, followed by $4 \%$ paraformaldehyde solution of $4^{\circ} \mathrm{C}$. The brains were removed and immersed in the $4 \%$ formaldehyde solution overnight at $4{ }^{\circ} \mathrm{C}$. Afterwards, the brains were processed through graded alcohols and xylene. By using a brain matrix, the forebrain was cut coronally into two equally spaced slices and embedded in paraffifin blocks after detaching the hindbrain. The brain sections located at $1.5 \mathrm{~mm}$ behind the bregma in the coronal plane were cut into $5 \mathrm{~mm}$ sections with vibratome (Leica, Solms, Germany) and mounted on the glass slides. The sections were deparaffifinized in xylene and rehydrated in $100-70 \%$ gradient ethanol. Finally, they were stained with hematoxylin-eosin (H\&E staining). The slices were washed with double distilled water, dehydrated in ethanol, cleaned with xylene, and examined with light microscopy. The pictures were taken with a digital camera. The sections corresponding to the Plate 35 contained the brain atlas were selected for analysis. The images were captured using the computer assisted image analyzer system consisting of a microscope (Olympus BX-51, Tokyo, Japan) magnifification at $\times 100$.

\subsection{Western blot analysis}

Western blot analysis was performed as previously described[34]. Animal tissues were homogenized and centrifuged at $4^{\circ} \mathrm{C}$. Protein concentrations in the supernatant were determined using a BCA protein assay reagent kit (Novagen, Madison, WI, USA) with bovine serum albumin (BSA) as the standard. Equal amounts of protein $(30 \mu \mathrm{g})$ were separated using $10 \%$ SDS-PAGE and subsequently transferred to PVDF membranes(Merck Millipore, Billerica, MA, USA) using electrophoresis. The membranes were blocked with $5 \% \mathrm{BSA}$ for $2 \mathrm{~h}$ and then incubated overnight at $4{ }^{\circ} \mathrm{C}$ with primary antibodies against ZO-1 (1:800, Abcam, Cambridge, MA, USA), claudin-5 (1:800, Abcam), occludin (1:800, Abcam), PPARy (1:800, Proteintech, Wuhan, China), NF-kB p65 (1:1,000, CST, Boston, MA, USA), phospho-NF-kB p65 (Ser536) (1:1,000, CST), and GAPDH (1:8,000, Bioworld, Louis Park, MN, USA) followed by incubation with peroxidase-conjugated secondary antibodies (1:8,000, Bioworld) and visualization using enhanced chemiluminescence (ECL) (Vazyme Biotech, Nanjing, China). The results were quantified using Image Lab software version 4.1 (BioRad, Hercules, CA, USA). GAPDH was used as the internal control.

\subsection{Immunofluorescence staining}


Mice ( $n=3$ per group) were anesthetized with $3 \%$ pentobarbital sodium $(0.1 \mathrm{~mL} / 10 \mathrm{~g}$ body weight), and transcardially perfused with $100 \mathrm{~mL}$ of normal saline, and then with $4 \%$ PFA in $100 \mathrm{~mL}$ of $0.1 \mathrm{M} \mathrm{PBS}(\mathrm{pH}$ = 7.4). Perfusion-fixed brains, lungs, and intestines were fixed in 4\% PFA in 0.1 M PBS overnight, cryoprotected with $40 \%$ sucrose, and sectioned at $20 \mu \mathrm{m}$ using a microtome (CM1950; Leica Microsystems, Wetzlar, Germany). The cryosections were washed three times for 5 min with PBS, blocked for $1.5 \mathrm{~h}$ in a blocking solution containing $5 \%$ BSA in PBS and $0.1 \%$ Triton X-100, and then incubated overnight at $4{ }^{\circ} \mathrm{C}$ with rabbit anti-mouse ZO-1 (1:200; ab96587), rabbit anti-mouse occludin (1:200; ab31721), rabbit anti-mouse claudin-5 (1:200; ab172968), and rabbit anti-mouse VE-cadherin (1:200; ab205336) antibodies (all from Abcam) diluted in blocking solution. After being rinsed three times with PBS, cryosections were incubated for $2 \mathrm{~h}$ with a fluorochrome-coupled secondary antibody (goat antirabbit; 1:200; Proteintech Group, Rosemont, IL, USA). Nuclei were stained with 4,6-diamidino-2phenylindole (DAPI) (Boyetime Institute of Biotechnology, Shanghai, China). After being rinsed with PBS, the cryosections were examined under a fluorescence microscope (Leica Microsystems)[34].

\subsection{Enzyme-linked immunosorbent assay (ELISA)}

The serum expression of TNF- $a$ and IFN- $y$ were detected using the corresponding ELISA kits (Yifeixue Bio Tech, Nanjing, China) according to the manufacturer's instructions. Absorption at $450 \mathrm{~nm}$ was determined using a microplate reader (Bio-Rad, Hercules, CA, USA), and TNF- $a$ and IFN- $y$ concentrations were determined according to the standard curve generated at the same time.

\subsection{Statistical analysis}

All the results are expressed as means \pm standard deviation. The Student's $t$-test was used for comparisons between two groups. One-way ANOVA was used for comparisons among three or more groups. All the tests were performed using GraphPad Prism v7 (GraphPad Software, La Jolla, CA, USA). Significance was established at $P<0.05$.

\section{Results}

\subsection{GRb1 ameliorated brain injury in MCAO/R mice}

As illustrated in Figure 1A, TTC staining showed that the area of ischemic infarction of the right brain was increased in the Model group compared with the Sham group, while infarction volumes in the GRb1 group were significantly reduced compared with those in the Model group. Edaravone, the positive control, also significantly reduced infarct volume at the dose of $3 \mathrm{mg} / \mathrm{kg}$. Representative samples of H\&E-stained brain sections are shown in Figure 1B. Mice in the Model group also displayed neuronal loss and shrunken cells in the right cerebral ischemic area, as well as a loss of nucleoli and wider perivascular space. In contrast, brain damage was significantly mitigated in the GRb1 and Eda groups. EB staining showed that there was a significant increase in EB leakage in the Model group when compared with that in the Sham group $(P<0.05)$. GRb1 and Eda treatment both led to a significant reduction in EB leakage $(P<0.05, P<$ 0.01) (Fig. 1C, D). 
3.2. GRb1 increased the expression of tight junction proteins in the brain after MCAO/R

To identify tight junction $(\mathrm{TJ})$ remodeling, the expression of ZO-1 and occludin were analyzed using immunofluorescence staining. Compared with the Model group, the average optical density for ZO-1 and occludin was significantly increased in the GRb1 group (Fig. 2A, B). The protein expression levels of ZO-1 and occludin were also determined by western blotting. The expression levels of both proteins were decreased in the Model group compared with the Sham group; whereas they were significantly increased in the GRb1 group compared with those in the Model group. (Fig. 2C, D).

\subsection{GRb1 ameliorated pulmonary injury in MCAO/R mice}

Quantitative spectrometric analysis showed extensive EB leakage in the lung after cerebral ischemia/reperfusion. EB leakage was significantly reduced in the GRb1 group when compared with the Model group $(P<0.05)$ (Fig. 3A). Pulmonary barrier injury was accompanied by an influx of neutrophils into the interstitium and bronchoalveolar space[35]. Neutrophil counts in alveolar lavage fluid were then determined, and were found to be significantly higher in the Model group than in the Sham group $(P<$ 0.05). The neutrophil content was markedly decreased in the GRb1 group, reaching levels similar to those of the Sham group (Fig. 3B). The results of H\&E staining showed that lung sections excised from mice in the Sham group were normal. In contrast, the alveolar space from animals in the Model group was wider, the alveolar cavity had shrunk, and normal alveolar tissue was lost. Interestingly, treatment with GRb1 greatly suppressed lung injury and also reduced histological damage (Fig. 3C).

\subsection{GRb1 increased the expression of junction proteins in the lung after MCAO/R}

To identify the effects of GRb1 on the pulmonary barrier, the expression of VE-cadherin and claudin-5 in the lung was analyzed by immunofluorescence staining. The results showed that the expression levels of both proteins were significantly decreased in the Model group compared with those in the Sham group, whereas in the GRb1 and the Eda groups they were increased (Fig. 4A, B). The expression of VE-cadherin and claudin- 5 in the lung was also determined by western blotting. The results showed that, the expression levels of these proteins in the GRb1 group were remarkably increased compared with the Model group $(P<0.05)$ (Fig. 4C, D).

\subsection{GRb1 ameliorated intestinal injury in MCAO/R mice}

To determine the effect of GRb1 on the intestinal barrier after cerebral I/R, an EB leakage assay was performed. The results showed that EB leakage was significantly increased $(P<0.05)$ in the Model group, whereas in the GRb1 group it was significantly decreased $(P<0.05)$ (Fig. 5A, B). The results of H\&E staining showed that, in mice of the Model group, the intestinal villi were shortened and thickened, the tips were broken, and neutrophils were infiltrated in the intestinal mucosa and submucosa. However, mice treated with GRb1 and Eda showed a marked improvement in intestinal injury compared with the Model group. The most severe intestinal injury was seen in the DSS group (Fig. 5C). Laser Doppler flowmetry is a practical and sensitive technique to evaluate the viability of the intestine[36]. Here, we used a Doppler 
blood flow analyzer to measure the mesenteric blood flow. Compared with the Sham group, the blood flow in the Model group was significantly decreased $(P<0.05)$; however, GRb1 treatment could extensively increase the blood flow compared with those in the Model group. $(P<0.05)$ (Fig. 5D).

\subsection{GRb1 increased the expression of tight junction proteins in the intestine after MCAO/R}

Considering that the tight junction proteins ZO-1 and occludin are critical for the maintenance of an intact intestinal permeability barrier, the effects of GRb1 on these proteins in the intestine after cerebral I/R were also examined. As expected, immunofluorescence staining results showed that, the expression levels of ZO-1 (Fig. 6A, left) and occludin (Fig. 6B, right) proteins were significantly decreased in the Model group compared with those in the Sham group, whereas in the GRb1 and the Eda groups they were increased compared with those in the Model group. The expression of ZO-1 and occludin in the intestinal epithelium was also determined by western blotting. The results demonstrated that the levels of both proteins were significantly increased in the GRb1 group compared with those in the Model group $(P<0.05)$ (Fig. 6C, D). These data suggested that the observed improvement in intestinal permeability was facilitated by the GRb1-mediated maintenance of tight junction protein levels.

\subsection{GRb1 improved brain/lung/intestinal barrier damage through the PPARY/NF-KB pathway}

The integrity of the BBB is known to be associated with PPARY/NF-KB signaling pathway[37]. In addition, GRb1 can reportedly increase intracellular PPARy activity[25]. To further validate the link between GRb1 and activation of PPARY/NF-KB pathway, the levels of PPARY and phospho-NF-KB p65 in the brain, lung, and intestinal tissues of mice were measured by western blotting. As shown in Figure 7B \& C, after cerebral I/R, the level of PPARY was significantly decreased in the Model group compared with the Sham group, whereas PPARy levels were significantly increased in the GRb1 group compared with those in the Model group $(P<0.05)$. In the brain, an increase in PPARy expression was observed in the Model group compared with the Sham group, however, GRb1 administration also significantly increased the levels of PPARY compared with those in the Model group (Fig. 7A). Stroke-induced NF-kB activation was markedly inhibited by GRb1 in the brain, lung and intestine compared with those in the Model group. Finally, the serum levels of inflammatory mediators, including TNF- $a$ and IFN- $\gamma$, were measured to evaluate the severity of inflammation. The levels of TNF-a and IFN- $\gamma$ were significantly lower in the GRb1 group than in the Model group $(P<0.05)$ (Fig. 7D)

3.8. The PPARy inhibitor, GW9662, abolished the protective effects of GRb1 on the different barriers in $\mathrm{MCAO} / \mathrm{R}$ mice

To investigate the essential role of PPARy in the three barriers, we administered GW9662, a PPARY inhibitor. Compared to the Model+GRb1 group, the Model+GRb1+GW group showed that GW9662 treatment reduced the integrity of the various barriers, as evidenced by the reduced expression of junction proteins in the brain, lung, and intestine (Fig. 8A). In addition, GW9662 administration also reduced the levels of PPARY, whereas those of phospho-NF-KB p65 were increased compared with the Model+GRb1 group (Fig. 8B). 


\section{Discussion}

In this study, we presented evidence that the permeability of the brain, lung, and intestinal barriers increased after stroke, and that GRb1 could effectively mitigate the stroke-induced damage to the three barriers. We also demonstrated that GRb1 exerted these effects through the PPARY/NF-KB signaling pathway, at least partly.

An increasing number of studies have shown that ischemic stroke causes a series of complications, including pneumonia and damage to the intestinal mucosa[38]. For instance, focal ischemic stroke has been associated with brain-lung crosstalk, leading to increased pulmonary damage and permeability of the pulmonary capillary membrane[39]. Additionally, intestinal mucosal barrier disturbance can be identified even in the early stages of cerebral ischemic stress. Necrosis occurs at the tip of the villi and the intestinal mucosa is damaged in the early stages of ischemic stroke, leading to gastrointestinal kinetic changes[40].

In our study, we found that both the pulmonary and intestinal barriers were simultaneously damaged in an experimental model of focal ischemic stroke. Several studies have demonstrated that GRb1 can be useful as a therapy for cardiovascular diseases[21], and exerts beneficial effects on the intestinal mucosal barrier[23] and lung inflammation[41]. However, its protective effects on brain/lung/intestinal barrier damage after ischemic stroke remain unclear.

In the present study, EB leakage was used to evaluate the integrity of the barriers of the three organs (brain, lung, and intestine). EB has a very high affinity for serum albumin, which cannot cross the blood barrier[42]. However, when a blood vessel is compromised, albumin, with bound EB, leaks out. Our findings demonstrated that treatment with GRb1 reduced EB leakage and ameliorated histopathological damage in the brain, lung, and intestine after cerebral I/R (Figs 1C, 3A, and 5A).

In the brain, a physical paracellular barrier is created by the presence of TJs between endothelial cells. TJs are the luminal-most cellular junctions and are composed of claudins, occludins, and zona occludens[43]. TJs are more often studied in terms of their role in the BBB, where they have been found to induce high transendothelial electrical resistance and reduce paracellular diffusion to a greater extent than adhesion junctions[44]. In our study, the expression of ZO-1 and occludin in brain endothelial cells was significantly higher in the GRb1 group than in the Model group (Fig. 2).

In the lung, junction protein levels were reduced in mice with cerebral I/R. Adherens junctions play a major role in the regulation of pulmonary barrier permeability[45], while the transmembrane protein VE-cadherin is a central player in adherens junction formation and the regulation of pulmonary barrier integrity. Moreover, lung barrier injury has been associated with decreased mRNA and protein levels of claudin5[46]. In our study, the expression of VE-cadherin and claudin-5 in the lung was significantly increased in the GRb1 group compared with that in the Model group (Fig. 4A, B). 
Patients with severe brain injury have been reported to experience reduced gastrointestinal motility. In our study, GRb1 administration led to a significant increase in mesenteric blood flow (Fig. 5D), indicating its potential efficacy for treating brain injury-induced gastrointestinal motility. Laboratory and clinical evidence have both indicated that the intestinal TJ-mediated barrier plays a critical role in the pathogenesis of intestinal and systemic diseases[47]. TJs encircle the apical ends of the lateral membranes of epithelial cells and determine the selectivity of their paracellular permeability to solutes[48,49]. In the current study, we showed that the levels of ZO-1 and occludin were significantly decreased after cerebral I/R, but increased after treatment with GRb1 or Eda. This indicated that GRb1 can help maintain TJ-mediated barrier integrity following stroke-induced barrier disruption (Fig. 6A, B). Combined, these findings implied that GRb1 could effectively mitigate brain, pulmonary and intestinal barrier dysfunction in mice with cerebral I/R.

There is clear evidence that human stroke causes multi-organ systemic disease through multiple mechanisms. After a stroke, the stimulation of the vagus nerve through nicotinic acetylcholine receptor a7 (nAChRa7) induces microglial activation, resulting in reduced alveolar macrophage phagocytic capability and leading to lung injury[50]. Meanwhile, systemic inflammation consequent to stroke leads to an increase in the release of inflammatory mediators such as interleukin (IL)- 6 and TNF-a, resulting in lung inflammation and alveolar-capillary injury[51]. There exists bidirectional communication and interaction between the gut and the brain[52]. This link is thought to be based on the brain-gutmicrobiota axis, and involves the autonomic nervous system (ANS), the hypothalamic-pituitary-adrenal (HPA) axis, and the gastrointestinal tract[53]. Moreover, inflammatory molecules and cellular debris, the so-called DAMPs, are released from damaged tissue after a stroke. The release of DAMPs and cytokines, as well as the activation of the vagus nerve, induces gut dysmotility, gut dysbiosis, and increased gut permeability, resulting in the translocation of intestinal bacteria[54].

PPARY is an important member of the nuclear receptor superfamily and predominantly expressed in adipocytes. However, PPARy is also expressed in vascular cells, and data generated have shown the great importance of this receptor in vascular barrier integrity [55]. Studies have suggested that PPARY activation can inhibit inflammation, thereby maintaining barrier integrity[56]. PPARy may reduce proinflammatory phenotypes via suppression of the AP-1 and NF-KB pathways[57]. PPARY agonists can also significantly reduce ischemia-triggered increases in cerebrovascular/BBB permeability in mice by suppression of proapoptotic microRNA-15a (miR-15a)[58]. Systematic administration of the PPARY agonist pioglitazone was shown to ameliorate DSS-induced colitis by helping to repair the intestinal mucosal barrier. Moreover, pioglitazone could also inhibit ERK and NF-KB phosphorylation, as well as upregulate the expression of the tight junction proteins ZO- 1 and claudin- 5 in a PPARy signalingdependent manner[59].

Increasing evidences have shown that PPARY plays a upstream signal of NF-KB in the anti-inflammatory process[60]. The direct impact of PPARY on NF-KB may be associated with its enzymatic properties. PPARY is an E3 ubiquitin ligase, which cooperates with E2 UBCH3. PPARy mediates the ubiquitination of Lys48 NF-kB p65, which leads to the proteolytic degradation of this NF-KB subunit[61]. Recent studies 
have reported that GRb1 binds to the PPARy ligand-binding domain as determined by surface plasmon resonance, which suggests that GRb1 may be able to activate PPARY[62], while polTIRFM analysis also indicated that GRb1 could bind to PPARy[25]. GRb1 has also been shown to exert anticalcification effects through the PPARY/Wnt/ $\beta$-catenin axis[63] and can promote the browning of 3T3-L1 adipocytes through the activation of PPARy[64]. Based on these observations, we assessed the protein levels of PPARY and phospho-NF-KB p65 in MCAO/R mice treated with GRb1. We found that GRb1 treatment upregulated PPARY levels and inhibited those of phospho-NF-KB p65, with a concomitant reduction in the concentrations of proinflammatory factors, thereby helping to maintain the integrity of the brain, lung, and intestinal barriers. Moreover, we also found that these effects were suppressed by cotreatment with the PPARY antagonist GW9662.

The vascular barrier maintains vascular and tissue homeostasis and modulates many physiological processes. The integrity of vascular barriers can be disrupted by a variety of soluble permeability factors, and changes in barrier function can result in multi-organ damage during disease progression[65]. Dysregulated vascular hyperpermeability can participate in the progression of many pathological states, such as rheumatoid arthritis, inflammatory bowel disease, asthma, acute lung injury, and cardiovascular diseases[66]. PPARy expression is highest in brown and white adipose tissues, the large intestine, and immune cells, but can also be found in various other tissues, including the muscle, pancreas, liver, small intestine, and kidney[67]. Activators of PPARy exert a broad spectrum of biological functions, such as regulating fatty acid metabolism, reducing inflammation, influencing immune cell balance, inhibiting apoptosis and oxidative stress, and improving endothelial function. Such pleiotropic activity makes them interesting therapeutic targets for the treatment of various conditions[68]. Therefore, the protective effects elicited by GRb1 on barrier damage in multiple organs via PPARy suggest that it may have potential as a therapeutic option for the treatment of vascular barrier-related diseases.

\section{Conclusion}

In summary, we demonstrated for the first time that treatment with GRb1 can activate PPARY, inhibit phospho-NF-KB p65 expression, and ameliorate cerebral, pulmonary, and intestinal barrier impairments in mice with MCAO/R. Our findings demonstrated that GRb1 exerts beneficial effects on multi-organ damage after ischemic stroke and may serve as a potential clinically translatable therapy during recovery from stroke.

\section{Abbreviations}

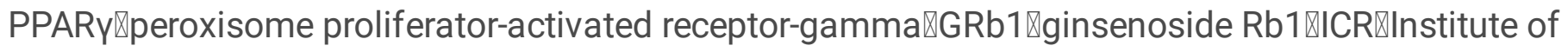

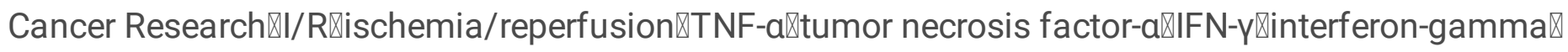

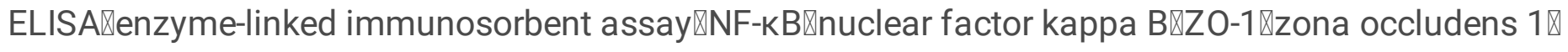

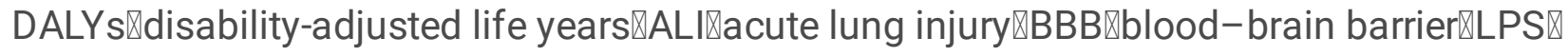

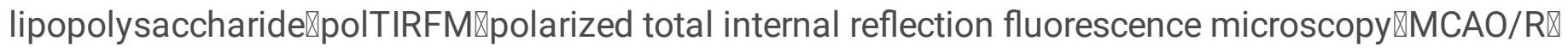




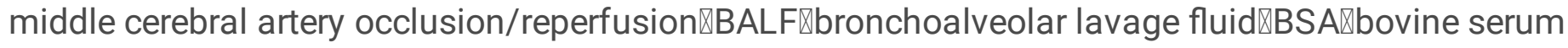
albumin囚H\&E囚hematoxylin-eosin.

\section{Declarations}

\section{Ethics approval and consent to participate}

Not applicable.

\section{Consent for publication}

Not applicable.

\section{Availability of data and materials}

The datasets used and/or analyzed during the current study are available from the corresponding author on reasonable request.

\section{Competing Interest}

The authors declare that they have no conflicts of interest.

\section{Funding}

This work was supported by the National Natural Science Foundation of China (No. 81773971) and "Double First-Class" University project (CPU2018GF07).

\section{Authors' contributions}

Junping Kou conceived and directed this research; Zhuo Chen carried out western blot experiments and mouse stroke model studies; Shuaishuai Gong and Yuchuan Ren carried out based biological studies; Jieman Wang provided technical support for drug labling study; Yuanyuan Zhang contributed material for this project; Fang Li wrote the manuscript.

\section{Acknowledgements}

Not applicable.

\section{References}

1. Hay SI, Abajobir AA, Abate KH, et al. Global, regional, and national disability-adjusted life-years (DALYs) for 333 diseases and injuries and healthy life expectancy (HALE) for 195 countries and territories, 1990-2016: A systematic analysis for the Global Burden of Disease Study 2016. Lancet. 2017;390:1260-1344. https://doi.org/10.1016/S0140-6736(17)32130-X. 
2. Naghavi M, Abajobir AA, Abbafati $C$, et al. Global, regional, and national age-sex specifc mortality for 264 causes of death, 1980-2016: A systematic analysis for the Global Burden of Disease Study 2016. Lancet. 2017;390:1151-1210. https://doi.org/10.1016/S0140-6736(17)32152-9.

3. Feigin VL, Krishnamurthi RV, Parmar P, et al. Update on the global burden of ischemic and hemorrhagic stroke in 1990-2013: The GBD 2013 study. Neuroepidemiology. 2015;45:161-176. https://doi.org/10.1159/000441085.

4. Santos SC, Pelosi P, Leme SP, et al. Immunomodulation after ischemic stroke: Potential mechanisms and implications for therapy. Crit Care. 2016;20:1-9. https://doi.org/10.1186/s13054-016-1573-1.

5. Chen Y, Li S, Sun F, et al. Monitoring of medical complications after acute ischemic stroke in a neurological intensive care unit. Eur Neurol. 2011;66:204-209. https://doi.org/10.1159/000330551.

6. Mascia L, Sakr Y, Pasero D, et al. Vincent, Extracranial complications in patients with acute brain injury: a post-hoc analysis of the SOAP study. Intensive Care Med. 2008;34:720-727. https://doi.org/10.1007/s00134-007-0974-7.

7. Bai W, Li W, Ning YL, et al. Blood glutamate levels are closely related to acute lung injury and prognosis after stroke. Front Neurol. 2018;8:755. https://doi.org/10.3389/fneur.2017.00755.

8. Elmer J, Hou P, Wilcox S, et al. Acute respiratory distress syndrome after spontaneous intracerebral hemorrhage. Crit Care Med. 2013;41:1992-2001. https://doi.org/10.1097/CCM.0b013e31828a3f4d.

9. Huang $Y, L i X, X$. Li, Sheng YY, et al. Neuroimmune crosstalk in central nervous system injury-induced infection and pharmacological intervention. Brain Res Bull. 2019;153:232-238. https://doi.org/10.1016/j.brainresbull.2019.09.003.

10. Camara-Lemarroy CR, Ibarra-Yruegas BE, Gongora-Rivera F. Gastrointestinal complications after ischemic stroke. J Neurol Sci. 2014;346:20-25. https://doi.org/10.1016/j.jns.2014.08.027.

11. Harari D, Norton C, Lockwood L, et al. Treatment of constipation and fecal incontinence in stroke patients: Randomized controlled trial. Stroke. 2004;35:2549-2555.

https://doi.org/10.1161/01.STR.0000144684.46826.62.

12. Crapser J, Ritzel R, Verma R, et al. Ischemic stroke induces gut permeability and enhances bacterial translocation leading to sepsis in aged mice. Aging (Albany. NY). 2016;8:1049-1063. https://doi.org/10.18632/aging.100952.

13. Yang $\mathrm{H}$, Gan $\mathrm{S}$, Jiang Z. et al. Protective effects of essential oil from Fructus Alpiniae zerumbet on retinal Müller gliosis via the PPAR-y-p-CREB signaling pathway. Chin Med. 2020;15:4. https://doi.org/10.1186/s13020-019-0283-4

14. Dong P, Zhao J, Li N, et al. Sevoflurane exaggerates cognitive decline in a rat model of chronic intermittent hypoxia by aggravating microglia-mediated neuroinflammation via downregulation of PPAR-y in the hippocampus. Behav Brain Res. 2018;347:325-331. https://doi.org/10.1016/j.bbr.2018.03.031.

15. Lin $\mathrm{MH}$, Chen $\mathrm{MC}$, Chen $\mathrm{TH}$, et al. Magnolol ameliorates lipopolysaccharide induced acute lung injury in rats through PPAR- $\gamma$-dependent inhibition of NF-kB activation. Int Immunopharmacol. 2015;28:270278. https://doi.org/10.1016/j.intimp.2015.05.051. 
16. Simeoli R, Raso GM, Lama A, et al. Preventive and therapeutic effects of lactobacillus paracasei B21060-based synbiotic treatment on gut inflammation and barrier integrity in colitic mice. J Nutr. 2015;145:1202-1210. https://doi.org/10.3945/jn.114.20598.

17. Rastogi V, Santiago-Moreno J, Doré S. Ginseng: A promising neuroprotective strategy in stroke. Front Cell Neurosci. 2015;8:1-13. https://doi.org/10.3389/fncel.2014.00457.

18. Yue PYK, Mak NK, Cheng YK, et al. Pharmacogenomics and the Yin/Yang actions of ginseng: antitumor, angiomodulating and steroid-like activities of ginsenosides. Chin Med. 2007;2:6. https://doi.org/10.1186/1749-8546-2-6.

19. Liu J, Liu Y, Wang Y, et al. GC-MS metabolomic analysis to reveal the metabolites and biological pathways involved in the developmental stages and tissue response of panax ginseng. Molecules. 2017;22:496. https://doi.org/10.3390/molecules22030496.

20. Yao F, Yang J, Huang Y, et al. Antinociceptive effects of Ginsenoside Rb1 in a rat model of cancer-induced bone pain. Exp Ther Med. 2019;17:3859-3866. https://doi.org/ 10.3892/etm.2019.7404.

21. Jiang N, Jing L, Wang $H$, et al. Ginsenoside 20(S)-protopanaxadiol attenuates depressive-like behaviour and neuroinflammation in chronic unpredictable mild stress-induced depressive rats. Behav Brain Res. 2020;393:112710. https://doi.org/10.1016/j.bbr.2020.112710S.

22. Yuan Q, Jiang YW, Ma TT, et al. Attenuating effect of Ginsenoside Rb1 on LPS-induced lung injury in rats. J. Inflamm. (United Kingdom). 2014;11:40. https://doi.org/10.1186/s12950-014-0040-5.

23. Zhou F, Zhang P, Chen $X$, et al. Ginsenoside Rb1 protects the intestinal mucosal barrier following peritoneal air exposure. Exp Ther Med. 2016;12:2563-2567. https://doi.org/10.3892/etm.2016.3639.

24. Chan LS, Kok TW, Keung MH, et al. Ginsenoside-Rb1 promotes adipogenesis through regulation of PPARy and microRNA-27b. Horm Metab Res. 2012;44:819-824. https://doi.org/10.1055/s-00321321909.

25. Kwok KC, Cheung NH. Measuring binding kinetics of ligands with tethered receptors by fluorescence polarization and total internal reflection fluorescence. Anal Chem. 2010;82:3819-3825. https://doi.org/10.1021/ac1002245.

26. Longa EZ, Weinstein PR, Carlson S, et al. Reversible middle cerebral artery occlusion without craniectomy in rats. Stroke. 1989;20:84-91. https://doi.org/10.1161/01.STR.20.1.84.

27. Cai Q, Xu G, Liu J, et al. A modification of intraluminal middle cerebral artery occlusion/reperfusion model for ischemic stroke with laser Doppler flowmetry guidance in mice. Neuropsychiatr Dis Treat. 2016;12:2851-2858. https://doi.org/10.2147/NDT.S118531.

28. Park DD, Yum HW, Zhong $X$, et al. Perilla frutescens extracts protects against dextran sulfate sodiuminduced murine colitis: NF-KB, STAT3, and Nrf2 as putative targets. Front Pharmacol. 2017;8:482. https://doi.org/10.3389/fphar.2017.00482.

29. Zhang $Y$, Xu T, Pan Z, et al. Shikonin inhibits myeloid differentiation protein 2 to prevent LPS-induced acute lung injury. Br J Pharmacol. 2018;175:840-854. https://doi.org/10.1111/bph.14129. 
30. Guan T, Liu Q, Qian Y, et al. Ruscogenin reduces cerebral ischemic injury via NF-kB-mediated inflammatory pathway in the mouse model of experimental stroke. Eur J Pharmacol. 2013;714: 303311. https://doi.org/10.1016/j.ejphar.2013.07.036.

31. Wang X, Kang K, Wang S, et al. Focal cerebral ischemic tolerance and change in blood-brain barrier permeability after repetitive pure oxygen exposure preconditioning in a rodent model. J. Neurosurg. 2016;125:943-952. https://doi.org/10.3171/2015.7.JNS142220.

32. Guo Z, Cao G, Yang H, et al. A combination of four active compounds alleviates cerebral ischemiareperfusion injury in correlation with inhibition of autophagy and modulation of AMPK/mTOR and JNK pathways. J Neurosci Res. 2014;92:1295-1306. https://doi.org/10.1002/jnr.23400.

33. Zetoune FS, Dhond RM, Canning BA, et al. During acute lung injury critical role for the NLRP3 inflammasome. J Immunol Ref. 2014;192:5974-5983. https://doi.org/10.4049/jimmunol.1400368.

34. Cao G, Chen H, Zhang Y, et al. YiQiFuMai Powder Injection ameliorates the oxygen-glucose deprivation-induced brain microvascular endothelial barrier dysfunction associated with the NF-KB and and ROCK1/MLC signaling pathways. J Ethnopharmacol. 2016;183:18-28. https://doi.org/10.1016/j.jep.2016.02.028.

35. Abraham E. Neutrophils and acute lung injury. Crit Care Med. 2003;31:S195-S199. https://doi:10.1097/01.CCM.0000057843.47705.E8.

36. Liu D, Svanberg K, Wang I, et al. Laser Doppler perfusion imaging: New technique for determination of perfusion and reperfusion of splanchnic organs and tumor tissue, Lasers. Surg. Med. 1997;20:473-479 https://doi.org/10.1002/(sici)1096-9101(1997)20:4<473::aid-Ism15>3.0.co;2-c.

37. Wu JS, Cheung WM, Hsu CY, et al. PPAR-y ameliorates neuronal apoptosis and ischemic brain injury via suppressing NF-kB-driven p22phox transcription. Mol Neurobiol. 2016;53:3626-3645. https://doi.org/10.1007/s12035-015-9294-z.

38. Wen SW, Wong CHY. An unexplored brain-gut microbiota axis in stroke. Gut Microbes. 2017;8:601606. https://doi.org/10.1080/19490976.2017.1344809.

39. Samary CS, Ramos AB, Maia LA, et al. Focal ischemic stroke leads to lung injury and reduces alveolar macrophage phagocytic capability in rats. Crit Care. 2018;22:249. https://doi.org/10.1186/s13054-018-2164-0.

40. Xu X, Zhu Y, Chuai J. Changes in serum ghrelin and small intestinal motility in rats with ischemic stroke. Anat Rec. 2012;295:307-312. https://doi.org/10.1002/ar.21490.

41. Lee J, Min D, Lee C, et al. Ginsenosides from Korean Red Ginseng ameliorate lung inflammatory responses: inhibition of the MAPKs/NF-KB/c-Fos pathways. J Ginseng Res. 2018;42:476-484. https://doi.org/10.1016/j.jgr.2017.05.005.

42. Hawkins B, Egleton R. Fluorescence imaging of blood-brain barrier disruption. J Neurosci Methods. 2006;151:262-267. https://doi.org/10.1016/j.jneumeth.2005.08.006.

43. Sweeney MD, Zhao Z, Montagne A, et al. Blood-Brain Barrier: from physiology to disease and back. Physiol Rev. 2019;99:21-78. https://doi.org/10.1152/physrev.00050.2017. 
44. Blanchette M, Daneman R. Formation and maintenance of the BBB. Mech Dev. 2015;138:8-16. https://doi:10.1016/j.mod.2015.07.007.

45. Lampugnani M, Dejana E, Giampietro C. Vascular endothelial (VE)-cadherin, endothelial adherens junctions, and vascular disease. Cold Spring Harb Perspect Biol. 2018;10:a029322. https://doi.org/10.1101/cshperspect.a029322.

46. Xia XM, Wang FY, Wang ZK, et al. Emodin enhances alveolar epithelial barrier function in rats with experimental acute pancreatitis. World J Gastroenterol. 2010;16:2994-3001. https://doi:10.3748/wjg.v16.i24.2994.

47. B L, M KM, K CY. Tight junction in the intestinal epithelium: its association with diseases and regulation by phytochemicals. J Immunol Res. 2018;2018:2645465. https://doi.org/10.1155/2018/2645465.

48. Tsukita S, Furuse M, Itoh M. Multifunctional strands in tight junctions. Nat Rev Mol Cell Biol. 2001;2:285-93. https://doi:10.1038/35067088.

49. Van Itallie CM, Anderson JM. Claudins and epithelial paracellular transport. Annu Rev Physiol. 2006;68:403-429. https://doi:10.1146/annurev.physiol.68.040104.131404

50. Cheyuo C, Jacob A, Wu R. The parasympathetic nervous system in the quest for stroke therapeutics. J Cereb Blood Flow Metab. 2011;31:1187-1195. https://doi:10.1038/jcbfm.2011.24.

51. Neumann S, Shields NJ, Balle T, et al. Innate immunity and inflammation post-stroke: an a7-nicotinic agonist perspective. Int J Mol Sci. 2015;16:29029-29046. https://doi:10.3390/ijms161226141.

52. Omran Y, Aziz Q. The brain-gut axis in health and disease. Adv Exp Med Biol. 2014;817:135-153. https://doi:10.1007/978-1-4939-0897-46.

53. Li XJ, You XY, Wang CY, et al. Bidirectional Brain-gut-microbiota Axis in increased intestinal permeability induced by central nervous system injury. CNS Neurosci Ther. 2020;26:783-790. https://doi: 10.1111/cns.13401.

54. Chamorro Á, Meisel A, Planas AM, et al. The immunology of acute stroke. Nat Rev Neurol. 2012;8:401-410. https://doi: 10.1038/nrneurol.2012.98.

55. Duan SZ, Usher MG, Mortensen RM. Peroxisome proliferator-activated receptor-gamma-mediated effects in the vasculature. Circ Res. 2008;102:283-294. https://doi: 10.1161/ClRCRESAHA.107.164384.

56. Li Q, Tian Z, Wang M, et al. Luteoloside attenuates neuroinflammation in focal cerebral ischemia in rats via regulation of the PPARү/Nrf2/NF-кB signaling pathway. Int Immunopharmacol. 2019;66:309-316. https:doi.org/10.1016/j.intimp.2018.11.044M.

57. Wang N, Verna L, Chen NG, et al. Constitutive activation of peroxisome proliferator-activated receptorgamma suppresses pro-inflammatory adhesion molecules in human vascular endothelial cells. J Biol Chem. 2002;277:34176-34181. https://doi: 10.1074/jbc.M203436200.

58. Yin KJ, Fan Y, Hamblin M, et al. KLF11 mediates PPARy cerebrovascular protection in ischaemic stroke, Brain. 2013;136:1274-1287. https://doi:10.1093/brain/awt002. 
59. Huang $Y$, Wang $C$, Tian $X$, et al. Pioglitazone attenuates experimental colitis-associated hyperalgesia through improving the intestinal barrier dysfunction. Inflammation. 2020;43:568-578. https://doi:10.1007/s10753-019-01138-3.

60. Linghu KG, Wu GP, Fu LY, et al. 1,8-Cineole ameliorates LPS-induced vascular endothelium dysfunction in mice via PPAR-y dependent regulation of NF-KB. Front Pharmacol. 2019;10:178. https://doi.org/10.3389/fphar.2019.00178.

61. Hou Y, Moreau F, Chadee K. PPARY is an E3 ligase that induces the degradation of NFKB/p65. Nat Commun. 2012;3:1300. https://doi.org/10.1038/ncomms2270.

62. Lu H, Zhou X, Kwok HH. Ginsenoside-Rb1 mediated anti-angiogenesis via regulating PEDF and miR33a through the activation of PPAR-y pathway. Front Pharmacol. 2017;8:783. https://doi.org/10.3389/fphar.2017.00783.

63. Zhou P, Zhang X, Guo M, et al. Ginsenoside Rb1 ameliorates CKD-associated vascular calcification by inhibiting the Wnt/ $\beta$-catenin pathway. J Cell Mol Med. 2019;23:7088-7098. https://doi:10.1111/jcmm.14611.

64. Mu Q, Fang X, Li X, et al. Ginsenoside Rb1 promotes browning through regulation of PPARY in 3T3-L1 adipocytes. Biochem Biophys Res Commun. 2015;466:530-535. https://doi:10.1016/j.bbrc.2015.09.064.

65. Wettschureck N, Strilic B, Offermanns S. Passing the vascular barrier: Endothelial signaling processes controlling extravasation. Physiol Rev. 2019;99:1467-1525. https://doi.org/10.1152/physrev.00037.2018.

66. Profaci CP, Munji RN, Pulido RS, et al. The blood-brain barrier in health and disease: Important unanswered questions. J Exp Med. 2020;217:1-16. https://doi.org/10.1084/jem.20190062.

67. Marion-Letellier R, Savoye G, Ghosh S. Fatty acids, eicosanoids and PPAR gamma. Eur J Pharmacol. 2016;785:44-49. https://doi.org/10.1016/j.ejphar.2015.11.004.

68. Vallée A, Lecarpentier Y. Crosstalk between peroxisome proliferator-activated receptor gamma and the canonical WNT/ $\beta$-catenin pathway in chronic inflammation and oxidative stress during carcinogenesis. Front Immunol. 2018;9:745. https://doi.org/10.3389/fimmu.2018.00745.

\section{Figures}


A

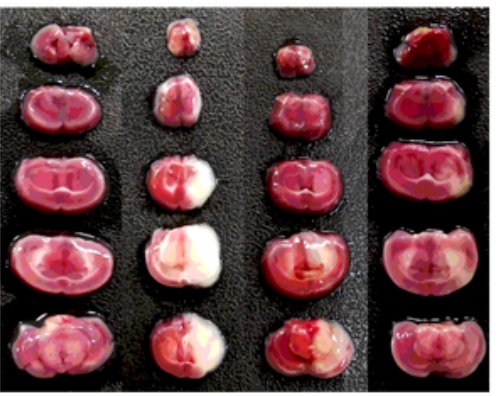

Sham

GRb1

Eda

C
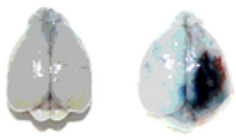

Model

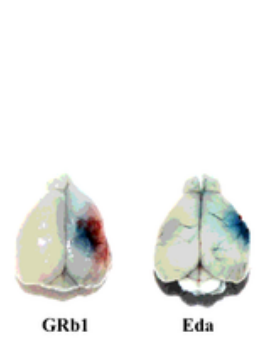

GRb1

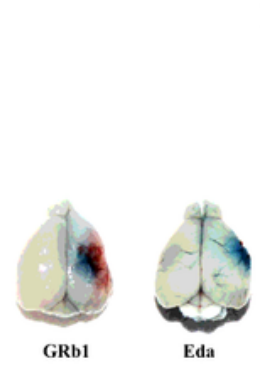

D
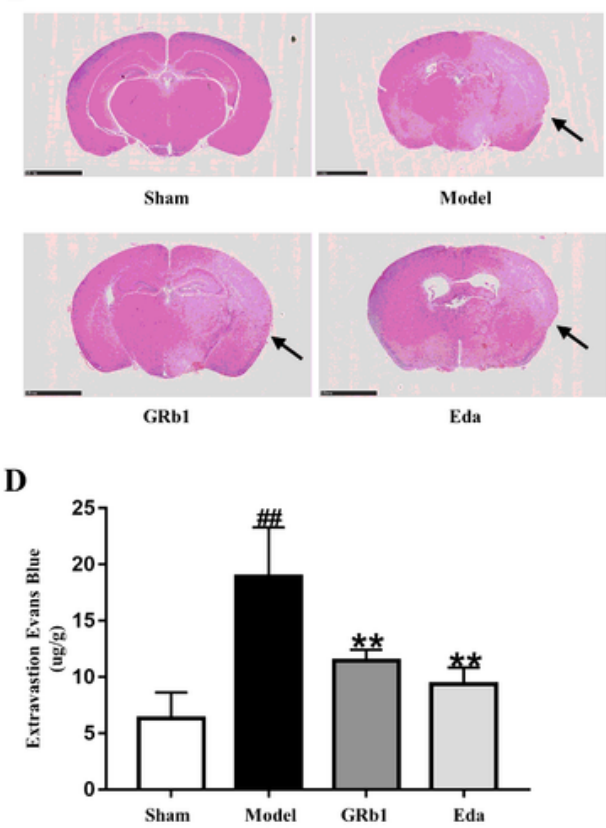

(1)

Sham Model GRb1 Eda

\section{Figure 1}

The effects of Ginsenoside Rb1 (GRb1) on brain injury in mice with cerebral ischemia/reperfusion (I/R) injury. (A) 2,3,5-Triphenyltetrazole ammonium chloride (TTC) staining in the brain. Red staining indicates normal brain tissue and the white (non-stained) part indicates infarcted tissue. (B) Hematoxylin and eosin staining of the brain sections of mice in the different groups. Scale bars $=2.5 \mathrm{~mm}$. Infarcted tissue is indicated by black arrowheads. (C) Representative images of Evans blue leakage in the brain tissues. (D) 
Quantitative analysis of EB extravasation by spectrophotometry. The data $(n=6)$ are expressed as means \pm SD. \#\# $P<0.01$ vs. the Sham group; $* P<0.05$, ** $P<0.01$ vs. the Model group.

A

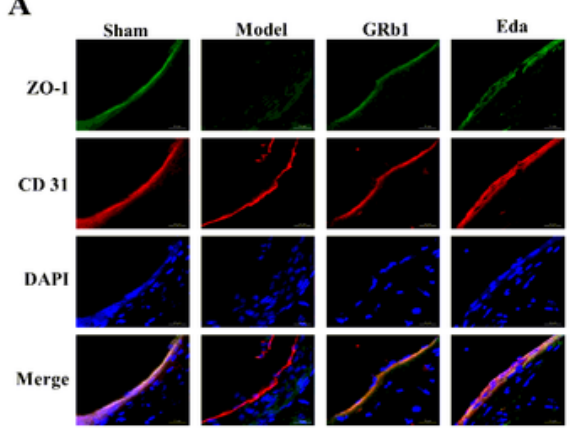

C
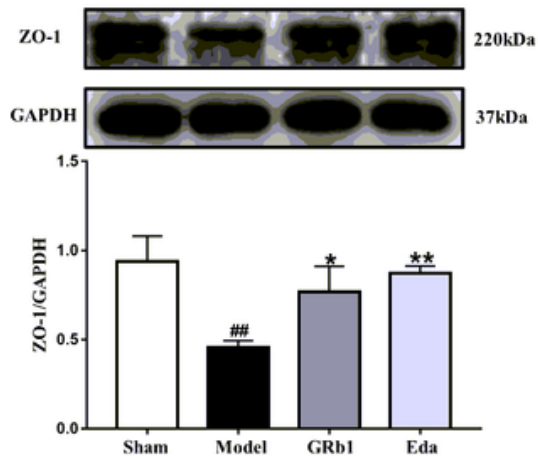

B

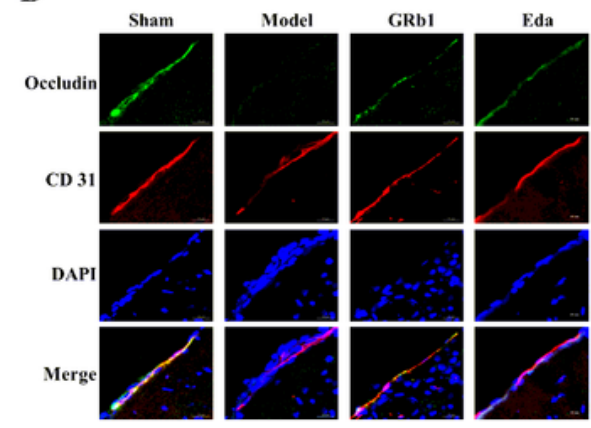

D

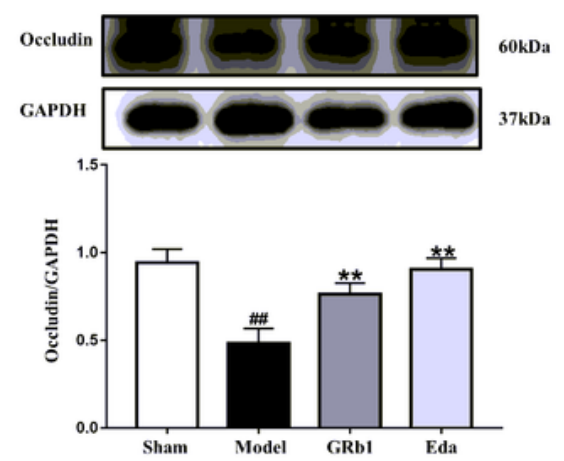

Figure 2

The effects of Ginsenoside Rb1 (GRb1) on the expression of tight junction proteins in the brain endothelium. (A, B) Representative immunofluorescence images of ZO-1 and occludin (green) localized with the marker CD31 (red) (left: ZO-1; right: occludin) at the periphery of endothelial cells. DAPI-stained 
nuclei are depicted in blue. Scale bars $=20 \mu \mathrm{m}$. (C, D) Representative western blots and quantitative analysis of the ratios of ZO-1 and occludin. The data $(n=3)$ are expressed as means \pm SD. \#\#P<0.01 vs. the Sham group; * $\mathrm{P}<0.05,{ }^{\star *} \mathrm{P}<0.01$ vs. the Model group.

A
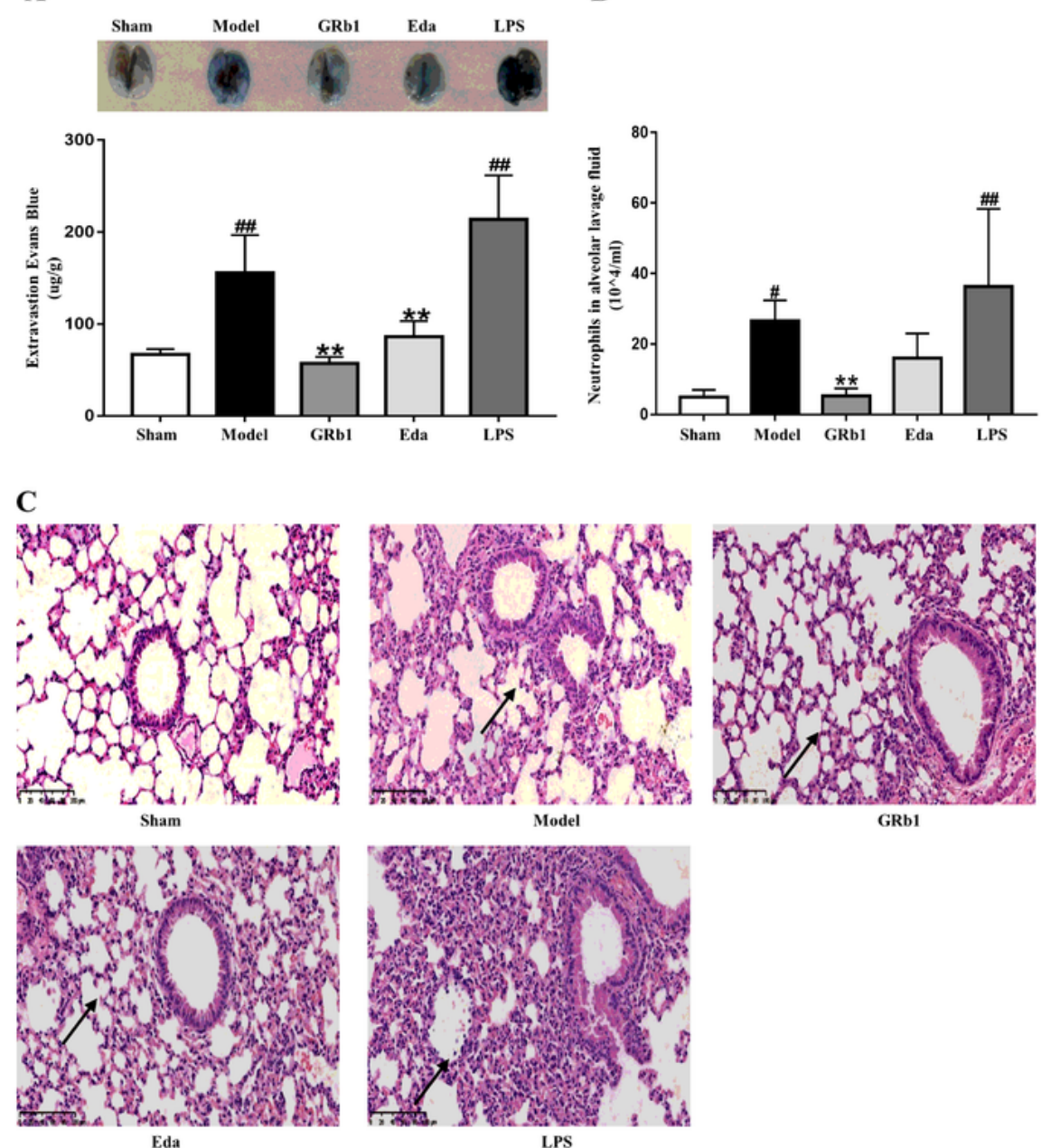

LPS

\section{Figure 3}

The effects of Ginsenoside Rb1 (GRb1) on lung injury in mice with cerebral ischemia/reperfusion (I/R). (A) Quantitative analysis of Evans blue leakage in the lung. (B) Determination of neutrophil numbers in 
alveolar lavage fluid. (C) The results of H\&E staining of lung tissue shows congestion in thick alveolar septa and alveolar collapse (black arrowheads). Scale bars $=100 \mu \mathrm{m}$. The data $(n=6)$ are expressed as means $\pm S D$. \# $P<0.05$, \#\# $P<0.01$ vs. the Sham group; * $P<0.05$, ** $P<0.01$ vs. the Model group.

$\mathbf{A}$

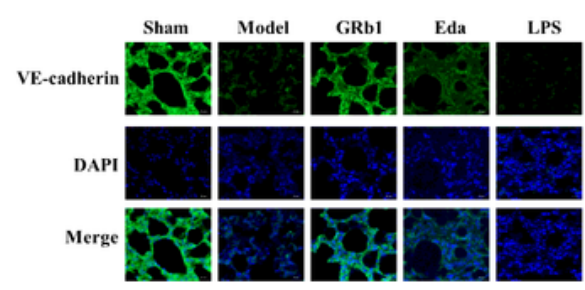

C
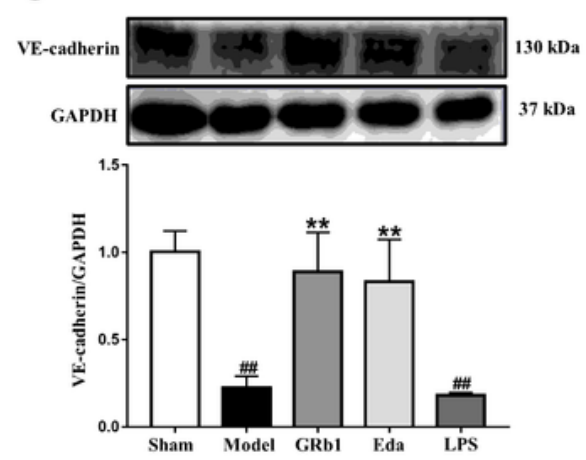

B

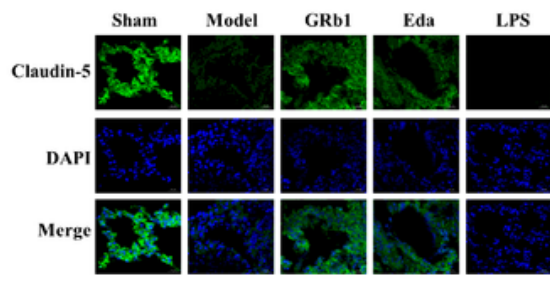

D
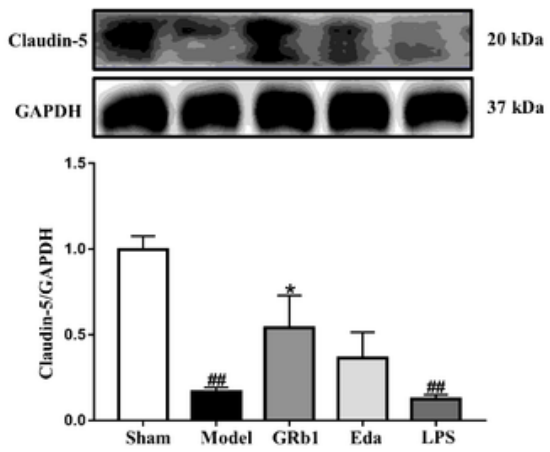

Figure 4

The effects of Ginsenoside Rb1 (GRb1) on the expression of junction proteins in the lung. (A, B) VEcadherin (left, A) and claudin-5 (right, B) are depicted in green, DAPI-stained nuclei are shown in blue. 
Scale bars $=20 \mu \mathrm{m}$. Representative western blots and quantitative analysis of the ratios of VE-cadherin (C) and claudin-5 (D). The data $(n=3)$ are expressed as means \pm SD. \#\# P< 0.01 vs. the Sham group; * $P$ $<0.05$, ** $\mathrm{P}<0.01 \mathrm{vs}$. the Model group.

A

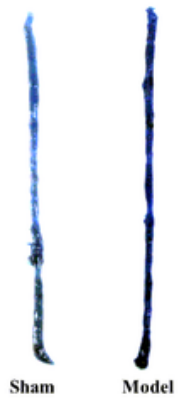

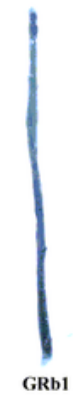

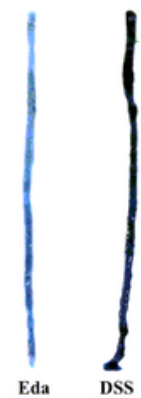

B

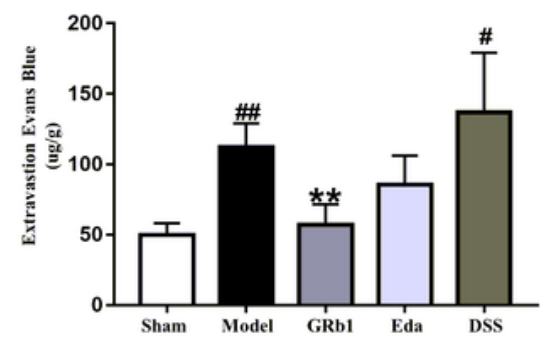

C
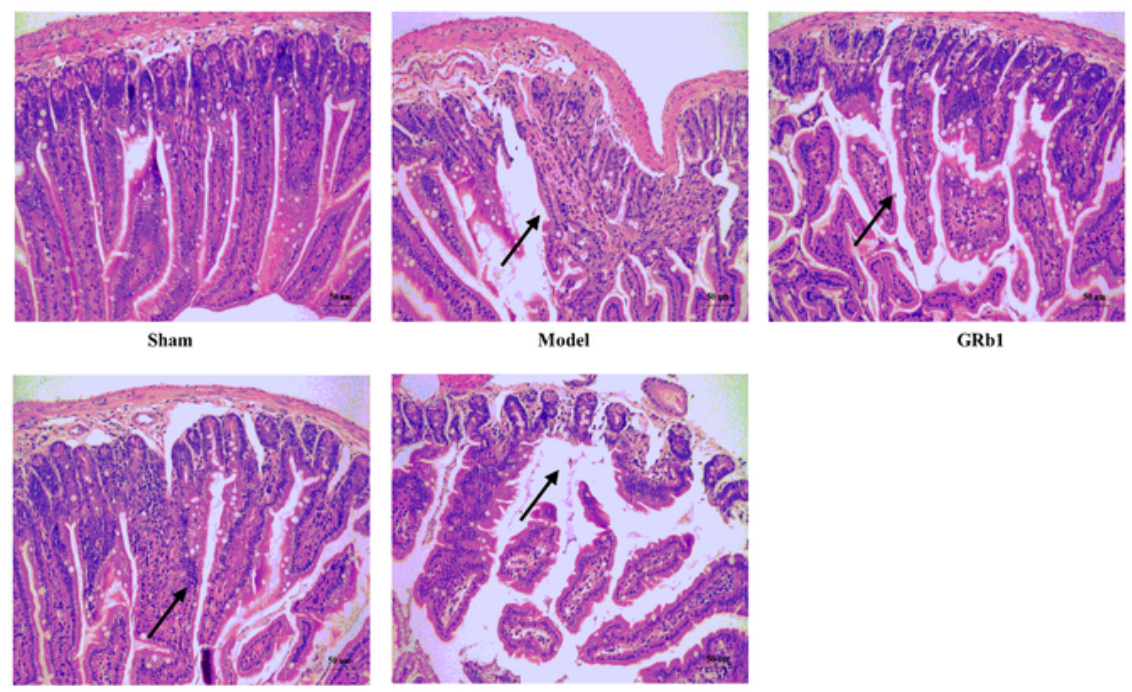

Eda

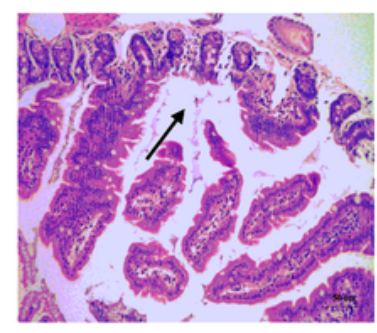

DSs

D

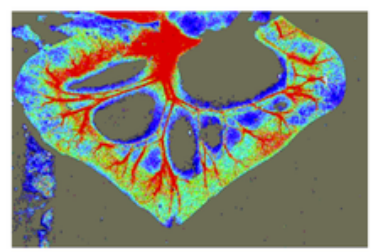

Sham

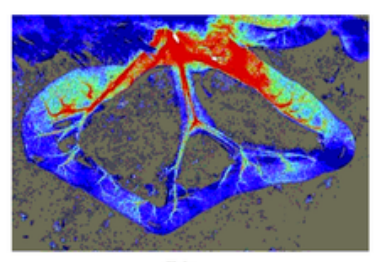

Eda

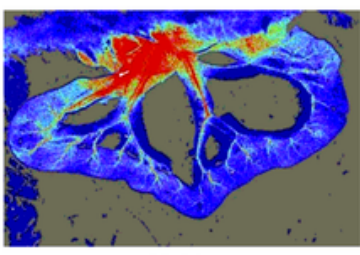

Model
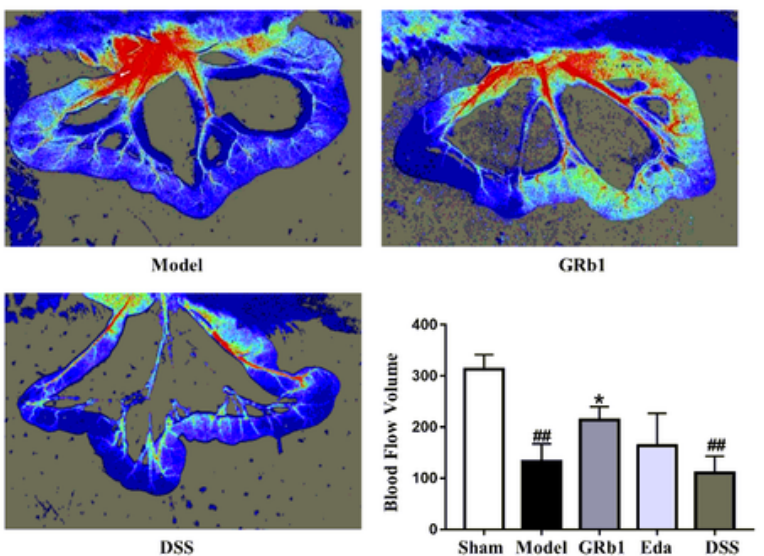

Figure 5

The effects of Ginsenoside Rb1 (GRb1) on intestinal injury in mice with cerebral ischemia/reperfusion (I/R). (A) Evans blue (EB) leakage in the intestine. (B) Quantitative analysis of EB extravasation by 
spectrophotometry. (C) Hematoxylin- and eosin-stained intestinal sections of mice in the different groups. Representative staining showing a gradual improvement in the morphology of the intestinal villi in the GRb1 group (black arrowheads). Scale bars $=50 \mu \mathrm{m}$. (D) Quantitative analysis of mesenteric blood flow in the different groups. The data $(n=6)$ are expressed as means $\pm S D$. \# $P<0.05$, \#\# $P<0.01$ vs. the Sham group; * $\mathrm{P}<0.05$, ** $\mathrm{P}<0.01$ vs. the Model group.
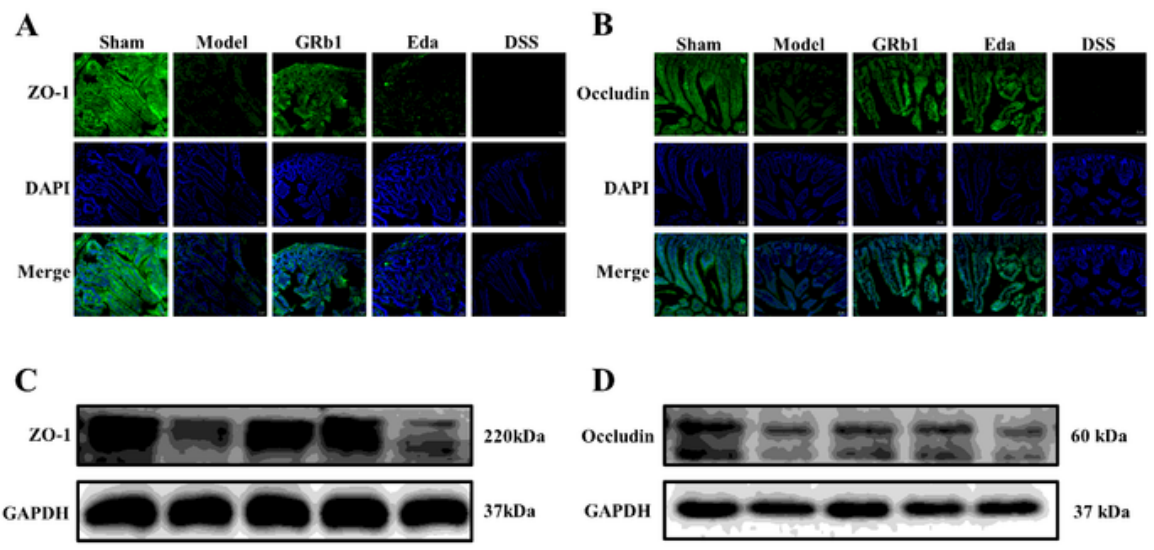

D
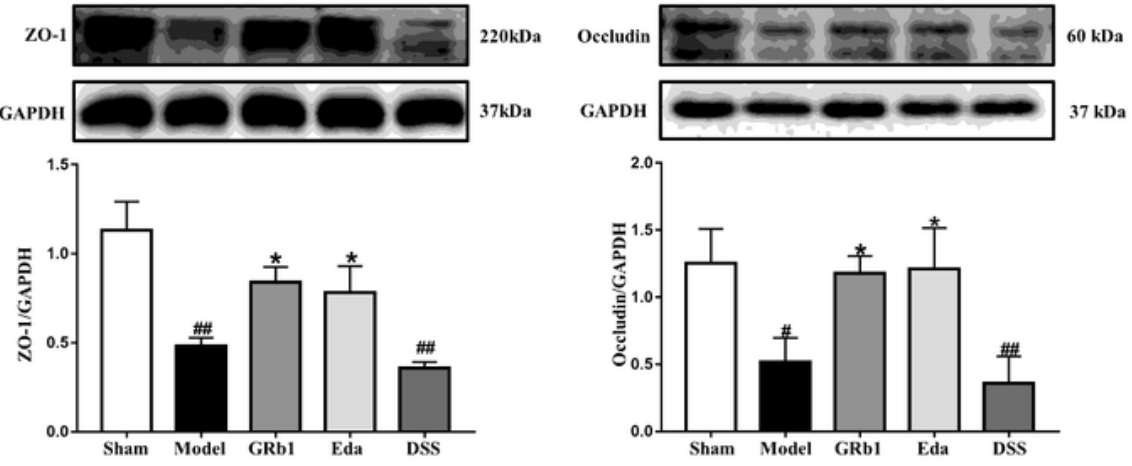

Figure 6 
The effects of Ginsenoside Rb1 (GRb1) on the expression of tight junction proteins in the intestine after cerebral ischemia/reperfusion (I/R). (A, B) ZO-1 (right) and occludin (left) are depicted in green and DAPIstained nuclei in blue. Scale bars $=20 \mu \mathrm{m}$. Representative western blots and quantitative analysis of the ratios of ZO-1 (C) and occludin (D). The data $(n=3)$ are expressed as means $\pm S D$. \# P< 0.05, \#\#P<0.01 vs. the Sham group; * $\mathrm{P}<0.05, * * P<0.01$ vs. the Model group.

$\mathbf{A}$

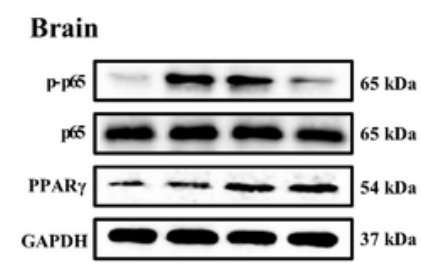

B
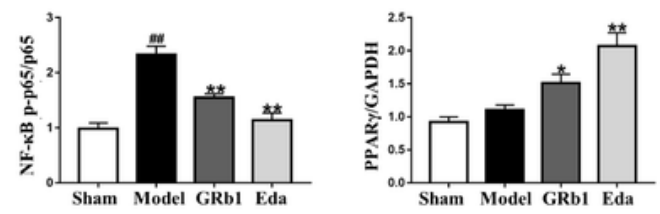

Lung

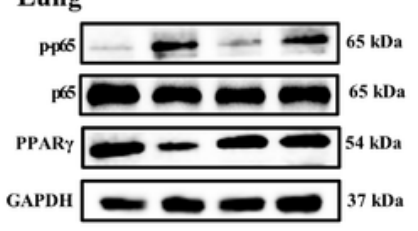

C
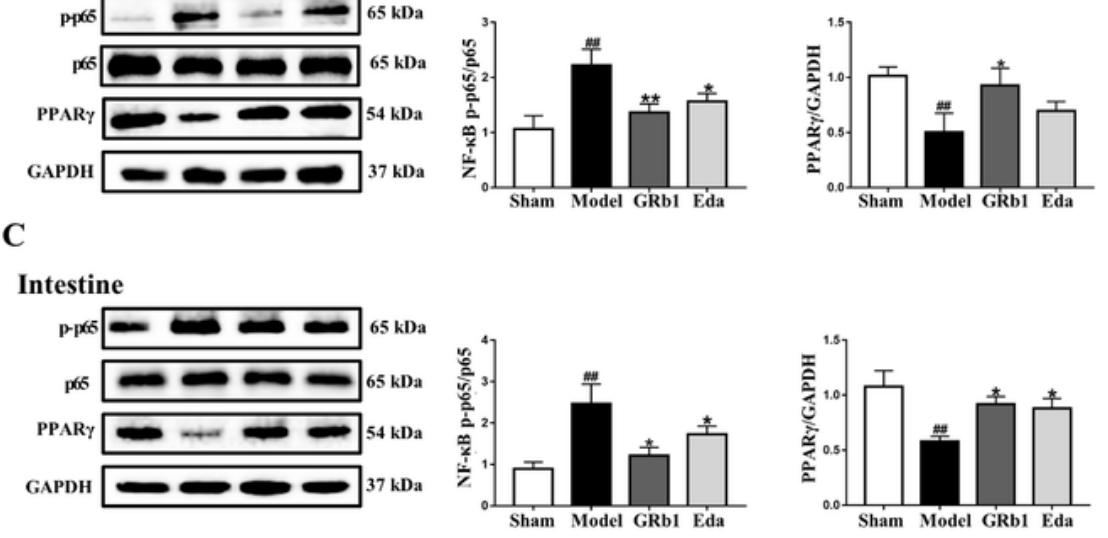

D
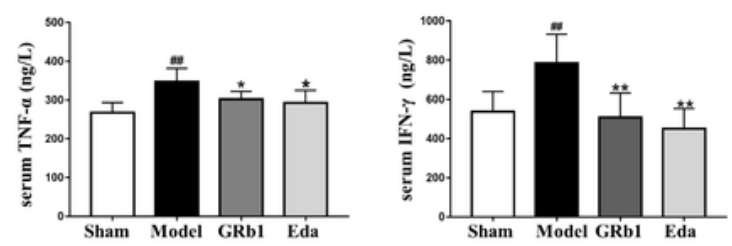

Figure 7 
The effects of Ginsenoside Rb1 (GRb1) on the PPARy/NF-KB signaling pathway and the levels of inflammatory mediators in mice with cerebral ischemia/reperfusion (I/R). The protein expression of PPARy, phospho-NF-KB p65 (Ser536), and NF-KB p65 in the brain (A), lung (B), and intestinal (C) tissue of mice was determined by western blots analysis ( $n=3$ /group). (D) Changes in the serum levels of TNF-a and IFN- $\gamma$ ( $n=6 /$ group). The data are expressed as means \pm SD. \# $P<0.05$, \#\# $P<0.01$ vs. the Sham group; ${ }^{\mathrm{P}}<0.05$, $* * \mathrm{P}<0.01$ vs. the Model group.
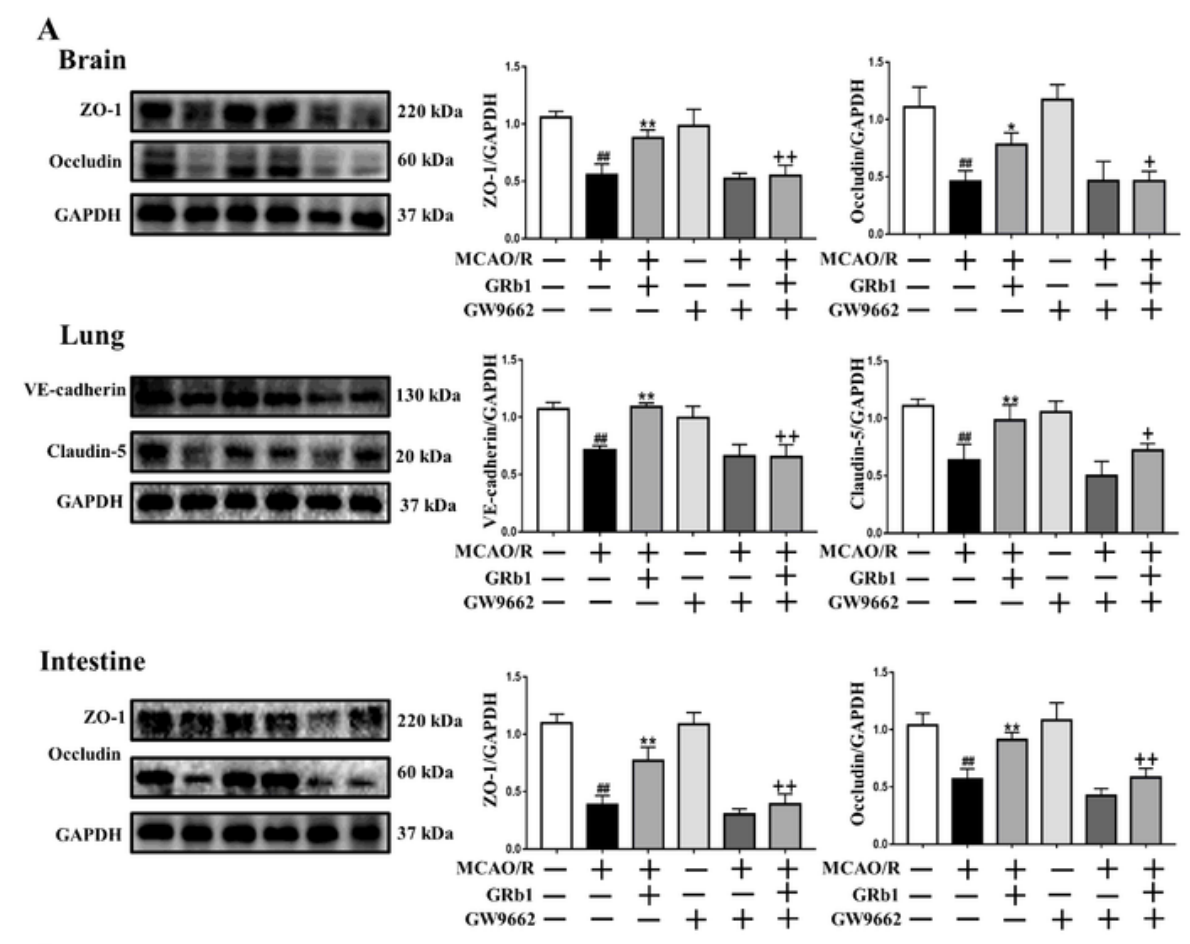

B

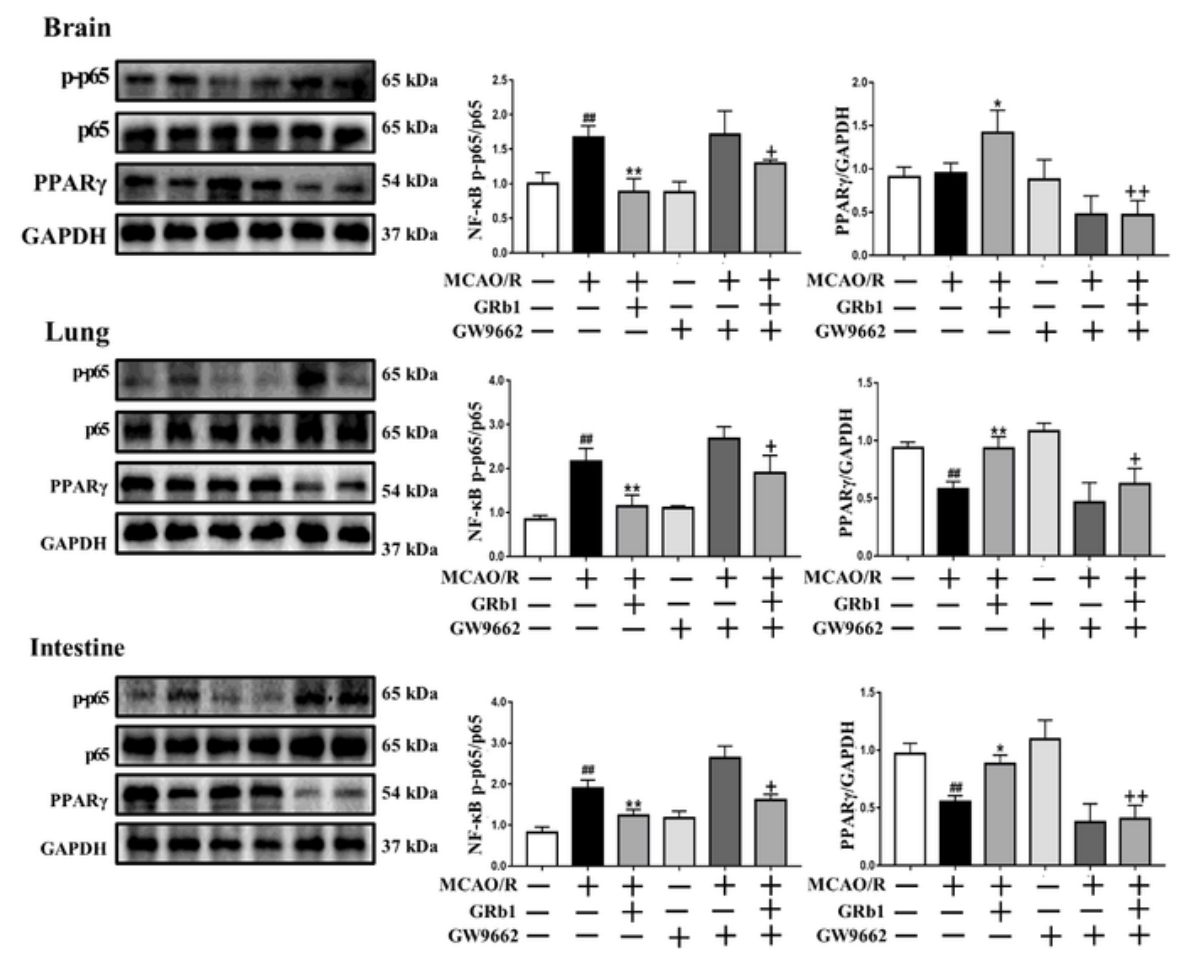

Figure 8 
GW9662 abrogated the effect of GRb1 on activation of PPARy/NF-KB signaling pathway in mice with cerebral ischemia/reperfusion (I/R). (A) Representative western blots and quantitative analysis of the ratios of ZO-1 and occludin in the brain, VE-cadherin and claudin-5 in the lung, and ZO-1 and occludin in the intestine. (B) The protein expression levels of PPARy, phospho-NF-kB p65 (Ser536), and NF-kB in the brain, lung, and intestine tissue of mice were determined by western blot $(n=3 /$ group). The data $(n=3)$ are expressed as means $\pm S D$. \# $P<0.05$, \#\# $P<0.01$ vs. the Sham group; $* P<0.05, * \star P<0.01$ vs. the Model group; $+\mathrm{P}<0.05,++\mathrm{P}<0.01$ vs. the Model+GRb1 group. 